\title{
Assessment of the Prevalence of HIV Infection at Four Health Facilities in Dilla Town, Ethiopia
}

Alemu $\mathrm{F}^{*}$

Department of Biology, College of Natural and Computational Sciences, Dilla University, Dilla, Ethiopia

*Corresponding author: Alemu F, Department of Biology, College of Natural and Computational Sciences, Dilla University, Dilla, Ethiopia, Tel: +251920839215, E-mail: fekealex@gmail.com

Citation: Alemu F (2015) Assessment of the Prevalence of Hiv Infection at Four Health Facilities in Dilla Town, Ethiopia. J Aids Hiv Infec 1(1): 101. doi: 10.15744/2454-499X.1.101

Received Date: February 05, 2015 Accepted Date: March 27, 2015 Published Date: April 07, 2015

\begin{abstract}
The successful introduction and spread of the human immunodeficiency virus (HIV) into the global human population may have first emerged among humans in the early part of the $20^{\text {th }}$ century (most likely contracted from infected primates). However its spread among vulnerables populations may have occurred in the 1970s. In Europe and North America the first cases of HIV/AIDS was identified among certain high risk groups' e.g. intravenous drug users, men having sex with men (MSM) etcs. HIV/AIDS is the common cause of death and morbidity among sexually active individuals in sub-Saharan Africa. The main objectives of this study was to assess the prevalence of HIV infection among patients attending four health centers in Dilla town from 2012-2014. Secondary data was obtained through assessing patients' records at these facilities. The number of HIV infected individual highest at 2012 followed by 2014 and 2013, 47, 37 and 30 respectively in Selam Higher Clinic while, the highest number of HIV infected was 31, followed by 29 and 19 in the year of 2013, 2014 and 2012 respectively at Fitsum Medium Clinic. On the other hand, the highest number of HIV infected was 38, followed by 33 and 15 in the year of 2013, 2014 and 2012 at Muluneh Higher Clinic and the number of HIV infected at Hikma Medium Clinic the highest was 7 at 2013 followed at 2012 and 2014 at Hikma Medium Clinic. Generally, distribution of HIV infected individuals at those clinics was slightly decreased across the year even if the number of the voluntary counseling tests was increase in these clinics. Majority of the people that live with HIV virus were in their productive age (15-49 age) among both male and female.

Keywords: HIV/AIDS; Prevalence; Dilla Town
\end{abstract}

\section{Introduction}

Acquired immunodeficiency syndrome has become one of the world's most serious health and development challenges [1]. Within Sub-Saharan Africa, the AIDS epidemic was noticed first in central Africa [2]. Soon after, the epidemic was observed in East Africa, and subsequently in West Africa [3]. There is a great deal of similarity between the HIV virus and an the human immunodeficiency virus is a retrovirus closely related to the Simian Immunodeficiency Virus (SIV) which is responsible for clinical immunodeficiency in other non-human primates especially the Afrcian green monkey, although the SIV does not cause immune-suppression among monkeys. This apparent correlation has led to speculation among scientists that African hunters who butchered and ate monkeys (a traditional food source) might have been exposed to a mutated form of the virus that was infective to humans [4]. The discovery of human immunodeficiency virus (HIV) (the virus responsible for acquired immunodeficiency syndrome (AIDS) was in 1983 and the explosion of the AIDS epidemic that brought clinical virology to the forefront as a significant specialty [5]. Viruses infect all cellular life forms: eukaryotes (vertebrate animals, invertebrate animals, plants, fungi) and prokaryotes (bacteria and archaea) [5].

Acquired Immune Deficiency Syndrome (AIDS) was first recognized as a new disease in 1981 when increasing numbers of young homosexual men succumbed to unusual opportunistic infections and rare malignancies [6,7]. By the mid-1980's, it became clear that the virus had spread, largely unnoticed, throughout most of the world [8]. The worldwide distribution of HIV/AIDS poses one of the biggest public health and social challenge in the world today [9]. According to the Joint UN Committee on HIV/AIDS [10], an estimated 38 million people worldwide were living with HIV in 2003, of which 5 million were newly infected. There are approximately 35 million people currently living with HIV and tens of millions of people have died of AIDS-related causes since the beginning of the epidemic [1]. While new cases have been reported in all regions of the world, approximately $68 \%$ are in subSaharan Africa [11]. Worldwide, women now represent $50 \%$ of all adults living with HIV and AIDS and this proportion had been steadily increasing over time [12].

The first case of AIDS in Ethiopia was reported in 1986 [13]. Other studies quoted 1984 (World Bank, 2004). Epidemics have since evolved into a generalized epidemic with AIDS-related death and opportunistic infections the leading cause of morbidity and mortality among adults (World Bank, 2004). Ethiopia has just over 1\% of the world's population but contributes $7 \%$ of the world's HIV/AIDS cases [14]. In terms of the number of people infected, it ranks fifth after South Africa, Nigeria, Kenya and Zimbabwe, 
and second (after Nigeria) in terms of the number of children orphaned by the AIDS epidemic [14]. More than 90\% of the infections in Ethiopia take place among those persons aged 15 to 49; the most economically productive segment of the population. The prevalence rate for 2004 was estimated at 13.2 for urban areas nationally and 2.3 for rural Ethiopia to give a national average of $3.7 \%$ [14].

The Ethiopian HIV/AIDS epidemic shares many features with that in Northeast and East Africa [15]. It is currently a general population epidemic, defined as more than $1 \%$ of the national sexually active adult's sero-positive, including more highly infected groups [15]. HIV-1 subtype C virus, is the major strain of HIV in Ethiopia and in most of Africa, which is thought to be associated with heterosexual transmission [16]. An estimated $87 \%$ of HIV transmission is through heterosexual contact, $10 \%$ by mother-tochild transmission and a smaller proportion is thought to be due to traditional harmful practices [17].

Soldiers, high-risk and mobile groups exposed to and spreading HIV through multi-partner sex contacts, were stationed in the 1980 s and early 1990s in many Ethiopian towns in the war zone. Troops were also at risk of being infected during emergency blood transfusions [18,19]. HIV infection rates in soldiers increased from $2.1 \%$ in 1985/86 to $12.0 \%$ in 1989 [20]. Harmful traditional practices, including female circumcision, violence against women and girls, including child marriage, rape, child sexual abuse, abduction and domestic violence, are widespread in Ethiopia. Female circumcision, which may be a risk factor for the transmission of HIV because of the bleeding and the sharing of infected sharps among different clients/individuals in some countries [21], was reported by 43 to $100 \%$ of Ethiopian women interviewed in different regions during the 2000 Demographic and Health Survey (DHS) [22].

In 2003, the highest HIV infection rates in Ethiopia reportedly occurred among persons 15-34 age groups. The highest rate among female ANC attendees was in the 15-24 age groups (8.6\%). Children and adolescents have become increasingly exposed to HIV in recent years, with an estimated 14,000 new infections among 0-14 age group in 2003 [23]. Rates are higher in young females than males, apparently due to a combination early sexual debut of females, the older age of their partners, gender-based biological factors [24], and prenatal and obstetric care/delivery exposures. In Ethiopia, in 2000, the median age of first sexual intercourse of women aged 20-49 was 16.4 years and for men 20.3 years, indicating the relatively greater vulnerability of teenage girls to HIV infection [22]. This study therefore assessed the prevalence of HIV infection in Dilla as well as the level of awareness of HIV among the local population of the town. In addition the finding helps to give possible baseline date for further study about the prevalence of HIV/AIDS in the study area. Therefore, this study was to assess the prevalence of HIV patient attending at Muluneh Higher Clinic, Selam Higher Clinic, Fitsum Medium Clinic and Hikma Medium Clinic clinic in Dilla town.

\section{Methdology of the Study}

\section{Description of the study area}

The study was conducted in Dilla town, it is found in Gedeo zone south west of the country and it located on the main road from Addis Ababa to Nairobi, the town is far from Addis Ababa $365 \mathrm{~km}$ South of Ethiopia. Based on 2005 cenesus of conducted by the central statistical agency of Ethiopia, Dilla has a total population of 61,114 whom 31,329 were men and 29,785 were women [25]. This study was conducted in Dilla town the following health facilities; Mulunh Medium Clinic, Selam Higher Clinic, Fitsum Medium Clinic and Hikma Medium Clinic in 2014.

\section{Data collection}

Secondary data were collected from organized data by refereeing secondary date from already recoded documents at these health facilities center, Dilla, Ethiopia. The data was obtained from patients' medical records.

\section{Ethical clearance}

The study protocol was reviewed and approved by Dilla University Ethical Clearance Committee. A written informed consent was obtained from each respondent before data collection.

\section{Statistical analysis}

Statistical analysis of the data was performed using SPSS statistical software (statistical package for Social Sciences for Windows SPSS, version 16.0), for the analysis of proportions and percentages at four Clinics found in the Dilla town. Secondary data was expressed through graphical and tabular.

\section{Results}

The results showed the HIV prevalence infection between the 2012-2014 at the four health facilities. There was a gender variation in prevalence between males and females. Majority of HIV-positives were in the economically productive age bracket (15-49 ages) among both genders (Table 1). 


\begin{tabular}{|c|l|l|l|l|}
\hline \multirow{1}{*}{ Sex } & \multirow{2}{*}{ Age category } & \multicolumn{3}{|c|}{ Years } \\
\cline { 3 - 6 } & & $\mathbf{2 0 1 2}$ & $\mathbf{2 0 1 3}$ & $\mathbf{2 0 1 4}$ \\
\hline \multirow{4}{*}{ Male } & $<15$ age & - & - & $1(2.70 \%)$ \\
\cline { 2 - 5 } & $15-49$ age & $22(46.81 \%)$ & $9(30.00 \%)$ & $15(40.55 \%)$ \\
\cline { 2 - 5 } & $>49$ age & $6(12.76 \%)$ & $1(3.33 \%)$ & $1(2.70 \%)$ \\
\cline { 2 - 6 } & Total & $28(59.57 \%)$ & $10(33.33 \%)$ & $17(45.95 \%)$ \\
\hline \multirow{4}{*}{ Female } & $<15$ age & - & - & - \\
\cline { 2 - 6 } & $15-49$ age & $19(40.43 \%)$ & $19(63.34 \%)$ & $19(51.35 \%)$ \\
\cline { 2 - 6 } & $>49$ age & - & $1(3.33 \%)$ & $1(2.70 \%)$ \\
\cline { 2 - 6 } & Total & $19(40.43 \%)$ & $20(66.67 \%)$ & $20(54.05 \%)$ \\
\hline
\end{tabular}

Table 1: Percentage of HIV infected patients at Selam Higher Clinic during 2014 year

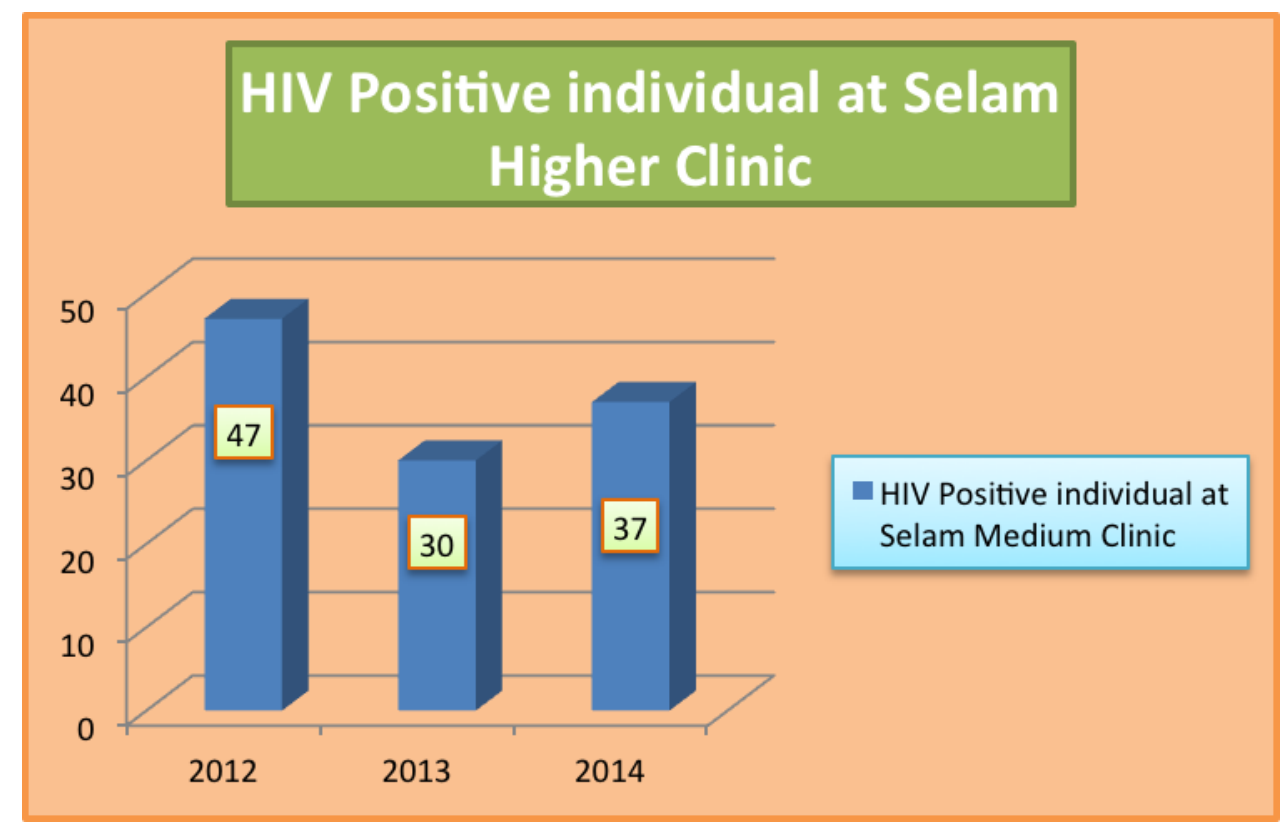

Figure 1: Distribution of HIV infected individual at Selam Higher Clinic

The number of HIV infected individual highest at 2012 followed by 2014 and 2013, 47, 37 and 30 respectively in Selam Higher Clinic as shown in Figure 1.

\begin{tabular}{|c|l|l|l|l|}
\hline \multirow{1}{*}{ Sex } & $\begin{array}{c}\text { Age category } \\
\text { (years) }\end{array}$ & \multicolumn{3}{|c|}{ Years } \\
\cline { 3 - 6 } & & $\mathbf{2 0 1 2}$ & $\mathbf{2 0 1 3}$ & $\mathbf{2 0 1 4}$ \\
\hline \multirow{4}{*}{ Male } & $<15$ & - & - & $1(3.85 \%)$ \\
\cline { 2 - 5 } & $15-49$ & $3(15.79 \%)$ & $6(19.35 \%)$ & $7(26.92 \%)$ \\
\cline { 2 - 6 } & $>49$ & $2(10.53 \%)$ & - & - \\
\cline { 2 - 6 } & Total & $5(26.32 \%)$ & $6(19.35 \%)$ & $8(30.77 \%)$ \\
\hline \multirow{4}{*}{ Female } & $<15$ & $1(5.26 \%)$ & - & $1(3.85 \%)$ \\
\cline { 2 - 6 } & $15-49$ & $13(68.42 \%)$ & $24(77.42 \%)$ & $17(65.38 \%)$ \\
\cline { 2 - 6 } & $>49$ & - & $1(3.23 \%)$ & - \\
\cline { 2 - 6 } & Total & $14(73.68 \%)$ & $25(80.65 \%)$ & $18(69.23 \%)$ \\
\hline
\end{tabular}

Table 2: Percentage of HIV infected at Fitsum Medium Clinic during 2014 year

HIV infected distribution in the age group15-49 years was higher among females than males while, across the year the infection was increase (Table 2). 


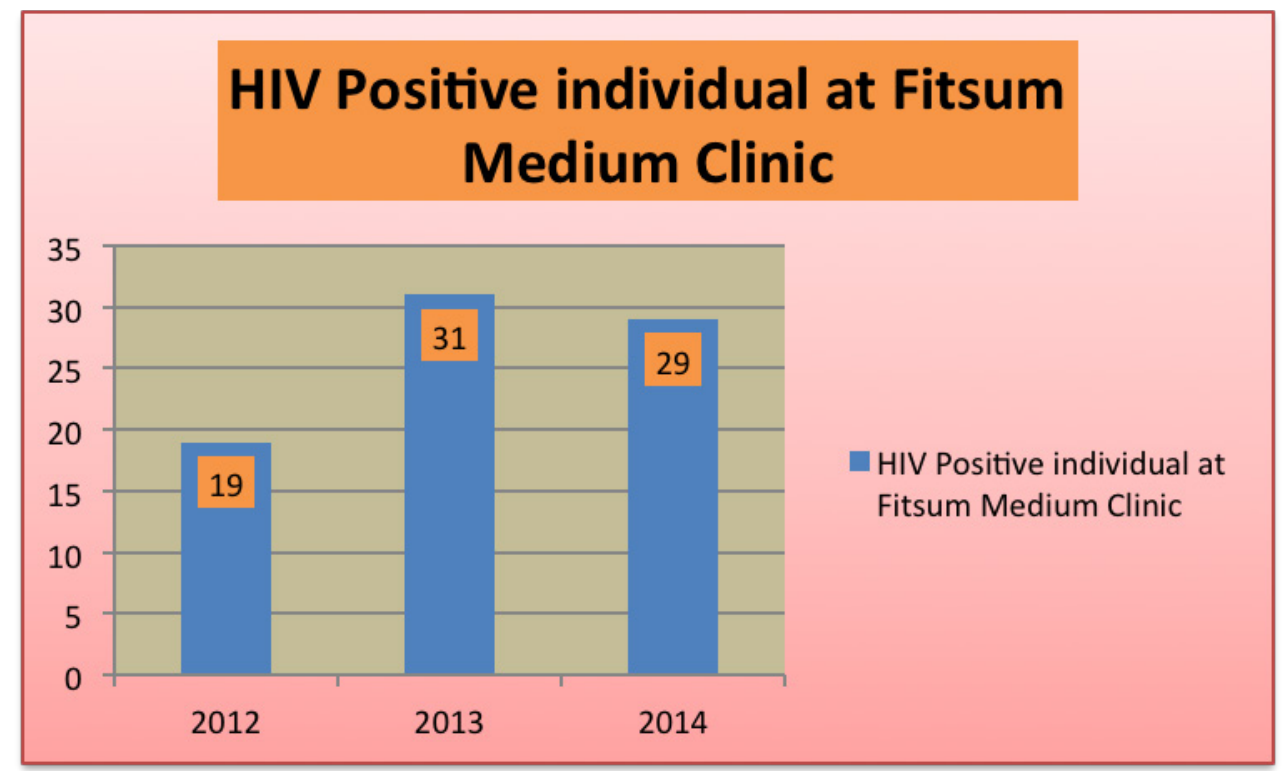

Figure 2: Distribution of HIV infected individual at Fitsum Medium Clinic

The highest number of HIV infected was 31, followed by 29 and 19 in the year of 2013, 2014 and 2012 respectively as shown in the (Figure 2).

\begin{tabular}{|c|l|l|l|l|}
\hline Sex & \multirow{2}{*}{ Age category } & \multicolumn{3}{|c|}{ Years } \\
\cline { 3 - 6 } & & $\mathbf{2 0 1 2}$ & $\mathbf{2 0 1 3}$ & $\mathbf{2 0 1 4}$ \\
\hline \multirow{5}{*}{ Male } & $<15$ years & - & - & - \\
\cline { 2 - 5 } & $15-49$ years & $\mathbf{6}(\mathbf{4 0 . 0 0} \%)$ & $20(52.63 \%)$ & $12(36.36 \%)$ \\
\cline { 2 - 5 } & $>49$ years & $1(6.67 \%)$ & $2(5.26 \%)$ & $2(6.06 \%)$ \\
\cline { 2 - 6 } & Total & $7(46.67 \%)$ & $22(57.89 \%)$ & $14(42.42 \%)$ \\
\hline \multirow{5}{*}{ Female } & $<15$ years & - & & - \\
\cline { 2 - 6 } & $15-49$ years & $8(53.33 \%)$ & $16(42.11 \%)$ & $19(57.58 \%)$ \\
\cline { 2 - 6 } & $>49$ years & - & - & - \\
\cline { 2 - 6 } & Total & $8(53.33 \%)$ & $16(42.11 \%)$ & $19(57.58 \%)$ \\
\hline
\end{tabular}

Table 3: Percentage of HIV infected at Muluneh Higher Clinic during 2014 year

HIV infected distribution among the age group15-49 years was across the sex more prevalent on the female than male, the highest rate at the year of 2013 (94.74\%) relatively with the other two years as indicated in Table 3.

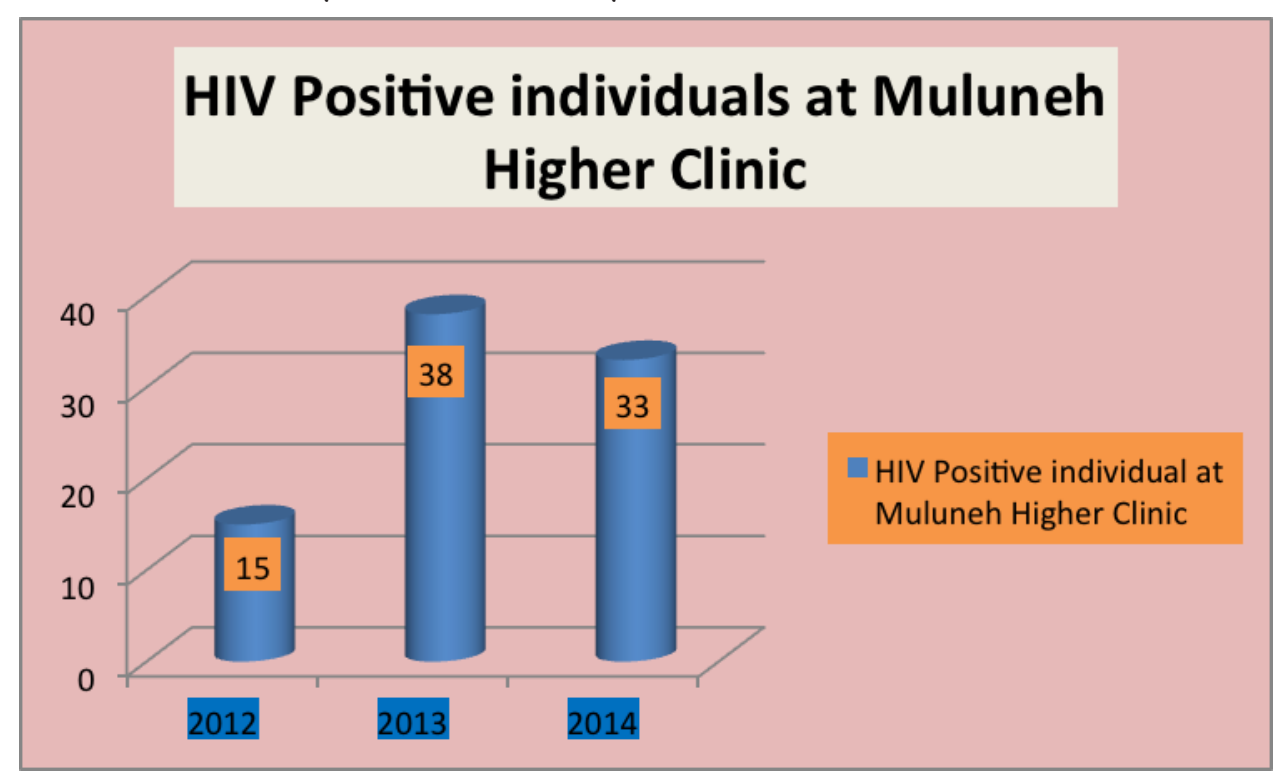

Figure 3: Distribution of HIV infected individual at Muluneh Higher Clinic

The highest number of HIV infected was 38, followed by 33 and 15 in the year of 2013, 2014 and 2012 respectively as shown in the (Figure 3). More than 95\% of HIV-infected people live in the developing world, most in Sub-Saharan Africa [26]. 


\begin{tabular}{|c|l|l|l|l|}
\hline \multirow{1}{*}{ Sex } & $\begin{array}{c}\text { Age category } \\
\text { (years) }\end{array}$ & \multicolumn{3}{|c|}{ Years } \\
\cline { 3 - 6 } & $\mathbf{2 0 1 2}$ & $\mathbf{2 0 1 3}$ & $\mathbf{2 0 1 4}$ \\
\hline \multirow{4}{*}{ Male } & $<15$ & - & - & $1(20.00 \%)$ \\
\cline { 2 - 5 } & $15-49$ & $3(60.00 \%)$ & $3(42.86 \%)$ & $1(20.00 \%)$ \\
\cline { 2 - 5 } & $>49$ & $1(20.00 \%)$ & - & - \\
\cline { 2 - 6 } & Total & $4(80.00 \%)$ & $3(42.86 \%)$ & $2(40.00 \%)$ \\
\hline \multirow{4}{*}{ Female } & $<15$ & - & $1(14.28 \%)$ & - \\
\cline { 2 - 6 } & $15-49$ & $1(20.00 \%)$ & $3(42.86 \%)$ & $3(60.00 \%)$ \\
\cline { 2 - 6 } & $>49$ & - & - & - \\
\cline { 2 - 6 } & Total & $1(20.00 \%)$ & $4(57.14 \%)$ & $3(60.00 \%)$ \\
\hline
\end{tabular}

Table 4: Proportion of HIV infected at Hikma Medium Clinic during 2014 year

HIV infected distribution in the age group 14-49 years was higher than the other age group. Therefore, the prevalence by age group and study period were; $\mathrm{s} 80.00 \%$ for $15-49$ years in 2012, $85.72 \%$ in 2013 and $80.00 \%$ in 2014 as shown in the Table 4 .

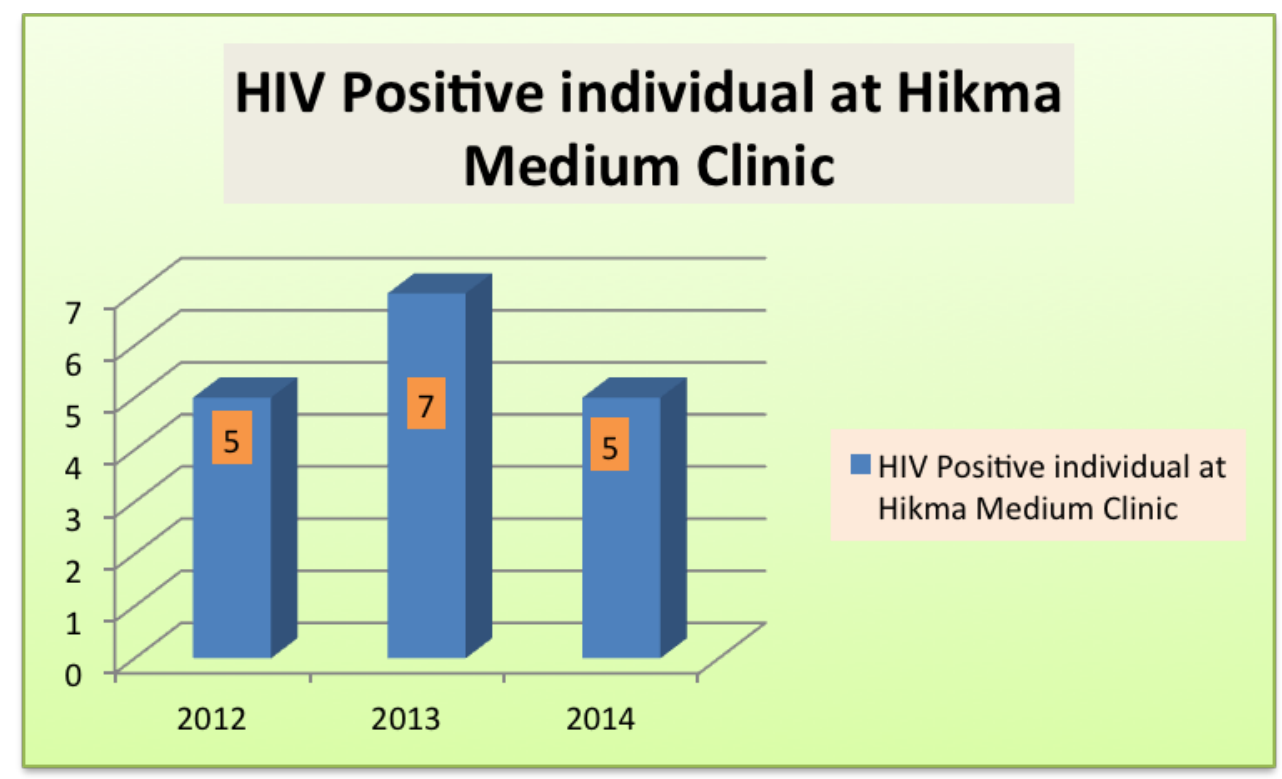

Figure 4: Distribution of HIV infected individual at Hikma Medium Clinic

The number of HIV infected at Hikma Medium Clinic the highest was 7 at 2013 followed by 2012 and 2014 as shown in the Figure 4.

\section{Discussion}

As the results shows prevalence of HIV infection was slightly higher among females than males in 2012 (Table 1). The seriousness of the HIV/AIDS epidemic in Ethiopia is widely acknowledged. Since the first HIV case was recorded in 1986, prevalence rates rose rapidly during the 1990s. By the end of 2003, it was estimated that 1.7 million people in the country (with a total population of over 70 million) had already died from AIDS and a further 1-2.3 million were living with the disease [27]. In addition, it is estimated that there are around 700,000 children under the age of 17 who have lost either one or both parents to AIDS.

The highest proportion of HIV infected was $96.77 \%$, followed by $92.30 \%$ and $84.21 \%$ in age-group of $15-49$ as shown in Table 2 . Ethiopia is classified (along with Nigeria, China, India, and Russia) as belonging to the "next wave countries" with large populations at risk from HIV infection, which will eclipse the current focal point of the epidemic in central and southern Africa [28]. Many developing countries that have a high prevalence of HIV infection also lack the resources to implement universal precautions adequately [29].

The prevalence by age group and study period were; s $80.00 \%$ for $15-49$ years in $2012,85.72 \%$ in 2013 and $80.00 \%$ in 2014 as shown in the Table 4. More than 95\% of HIV-infected people live in the developing world, most in Sub-Saharan Africa [26]. Improved data have revealed that the prevalence rates in southern Africa are staggering: $20-26 \%$ of adults (aged 15-49 yr) are infected; in some regions $20-50 \%$ of pregnant women are infected and are likely to transmit infection to one third of their offspring [26]. 
In 2003, the highest HIV infection rates in Ethiopia reportedly occurred in the 15-34 age groups. The highest rates in female ANC attendees were in the 15-24 age groups (8.6\%). Children and adolescents have become increasingly exposed to HIV in recent years, with an estimated 14,000 new infections in the 0-14 age group in 2003 [23]. In Ethiopia, in 2000, the median age of first sexual intercourse of women aged 20-49 was 16.4 years and for men 20.3 years, indicating the relatively greater vulnerability of teenage girls to HIV infection [22]. Globally, women now represent $50 \%$ of all adults living with HIV and AIDS and this proportion had been steadily increasing over time [12].

\section{Conclusion}

This study has shown that the prevalence of HIV/AIDS was more among females than males. There was a decline in prevalence of HIV infection at the four health facilities at Dilla between 2012-2014. This may be due to an increase in awareness about prevention in the community where the study was conducted.

\section{Acknowledgment}

I am grateful thanks to Dilla University, College of Natural and Computational sciences, Department of Biology who are giving these facilities to conduct this study. I extend also my thanks to Dilla town health facilities which are providing appropriate information for this study.

\section{References}

1. UNAIDS (2013) GAP Report.

2. Clumeck N, Mascart-Lemone F, de Maubeuge J, Brenez D, Marcelis L (1983) Acquired Immune Deficiency Syndrome in Black Africans. Lancet 1: 642.

3. Essex M, Mboup S (2002) Regional Variations in the African Epidemics. 631-40.

4. Countries and their Culture.

5. Carter JB, Saunders VA (2007) Virology: Principles and applications. John Wiley and Sons Ltd, England: 382.

6. Centers for Disease Control (CDC) (1981) Kaposi's sarcoma and Pneumocystis pneumonia among homosexual men-New York City and California. MMWR Morb Mortal Wkly Rep 30: 305-8.

7. Greene WC (2007) A history of AIDS: Looking back to see ahead. Eur J Immunol 37: 94-102.

8. UNAIDS and WHO (2003) A history of the HIV/aids epidemic with emphasis on Africa. Workshop on HIV/AIDS and Adult Mortality in Developing Countries New York.

9. Cai Y, Hong H, Shi R, Yi X, Li S, et al. ( 2008) Long term Follow up Study on Peer-led School Based HIV/AIDS Preventing among Youth in Shangi. Int J STD AIDS 19: 848-50.

10. UNAIDS (2004) AIDS epidemic update: Geneva, Switzerland, UNAIDS/WHO.

11. UNAIDS (2014) Epidemiological slides. GAP report 2014.

12. UNAIDS (2002) Report on the Global HIV/AIDS Epidemic. Addis Ababa: UNAIDS and WHO.

13. Hladik W, Shabbir I, Jelaludin A, Woldu A, Tsehaynesh M, et al. (2005) HIV/AIDS in Ethiopia: Where is the Epidemic Heading? Sexually Transmitted Infections 82: 32-5.

14. World Bank (2004) Ethiopia. A Country Status on Health and Poverty. Draft Report No.28963-ET. Africa Regional Human Development and Ministry of Health Ethiopia.

15. Kloos H, Mariam DH, Lindtjørn B (2007) The AIDS Epidemic in a Low-Income Country: Ethiopia. Human Ecology Review 14: 39-55.

16. Abebe A, Lukashov VV, Kliphuis TFA, Fontanet AI, Goudsmit G, et al. (2001) Timing of the HIV-1 subtype C epidemic in Ethiopia based on early virus strains and subsequent virus diversification. AIDS 15: 1555-61.

17. HAPCO (2004) Ethiopian Strategic Plan for Intensifying Multi-Sectoral HIV/AIDS Response. Addis Ababa, National HIV/AIDS Prevention and Control Office, Federal Ministry of Health.

18. Eshete H, Heast N, Lindan K, Mandel J (1993) Ethnic conflicts, poverty, and AIDS in Ethiopia. Lancet 341: 1219.

19. Kloos H (1993) Health impact of war. The Ecology of Health and Disease in Ethiopia 121-32.

20. Gebretensae GT (2003) HIV/AIDS in the Ethiopian military: perceptions, strategies, and impacts. Draft working paper for the CSIS Task Force on HIV/AIDS Committee on Destabilizing Impacts of HIV/AIDS. Addis Ababa.

21. de Walque D (2006) Who gets AIDS and how? The determinants of HIV infection and sexual behaviors in Burkina Faso, Cameroon, Ghana, Kenya, and Tanzania. World Bank Policy Research Working Paper WPS3844.

22. CSA and ORCMacro (2001) Ethiopia Demographic and Health Survey. Addis Ababa and Calverston, MA (USA), Central Statistical Authority and ORCMacro.

23. MOH (2004) AIDS in Ethiopia (5 $5^{\text {th }}$ edition) Addis Ababa: Ministry of Health.

24. Quinn TC, Overbaugh J (2005) HIV/AIDS in women: an expanding epidemic. Science 308: 1582-3.

25. CSA Statistical agency of Ethiopia.

26. Nancy A, Hessol MSPH, Monica Gandhi M.D.M.P.H, Ruth M, Greenblatt M.D (2005) A Guide to the Clinical care of women with HIV. Health Resources and Services Administration, HIV/AIDS Bureau.

27. UNAIDS, UNICEF, WHO (2004) Ethiopia, epidemiological fact sheets on HIV/AIDS and sexually transmitted infections. Geneva: WHO.

28. National Intelligence Council (2002) The next wave of HIV/AIDS: Nigeria, Ethiopia, Russia, India and China. Washington DC.

29. Gilks CF, Wilkinson D (1998) Reducing the risk of nosocomial HIV infection in British health workers working overseas: role of post-exposure prophylaxis. Br Med J 316: 1158-60. 


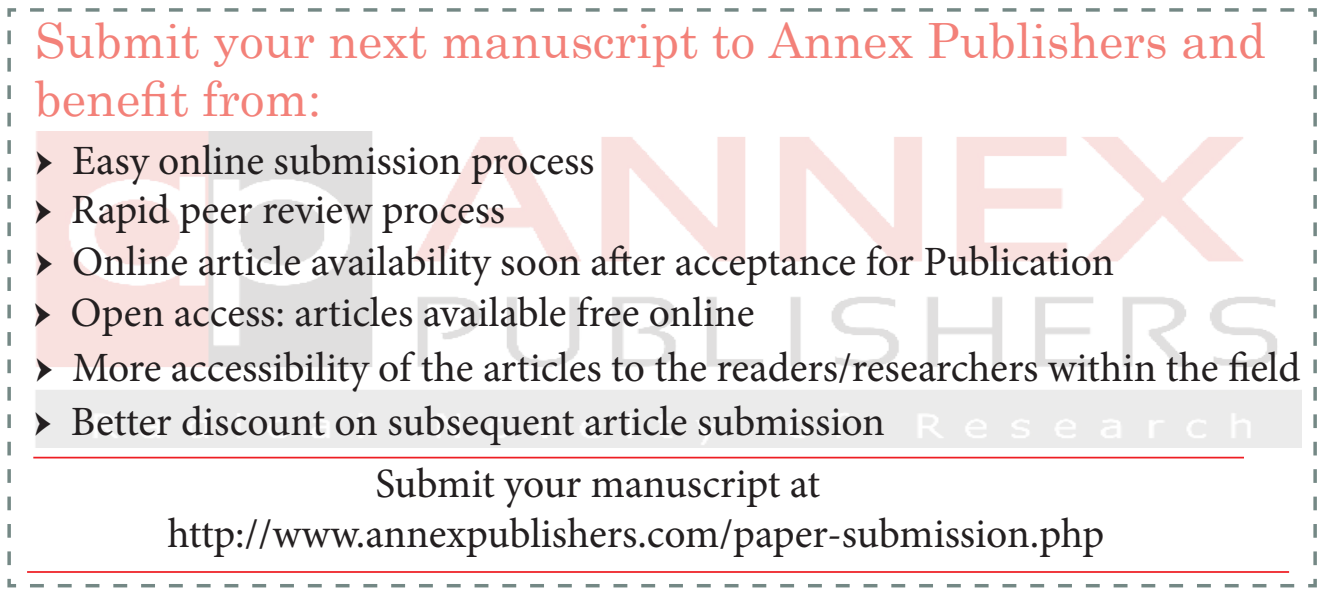

